Instrumental Achievements

\title{
On-Line Precolumn Derivatization Method for Determination of Amino Acids with o-Phthalaldehyde and 2-Mercaptoethanol
}

\author{
Qiuwen ZHAO* and Hiroko WADA** \\ * Tianjin University, Tianjin, China \\ **Nogoya Institute of Technology, Showa, Nagoya 466, Japan
}

Precolumn o-phthalaldehyde/2-mercaptoethanol (OPA/ MCE) derivatization methods have often been applied to the determination of amino acids by high performance liquid chromatography, because of their simplicity and high sensitivity. ${ }^{1}$ However, both the derivatization reagent (OPA/MCE) and the resultant isoindole derivatives degrade with time. Several automatic devices, therefore, were proposed for on-line precolumn derivatization ${ }^{2-4}$, but they need sophisticated instruments such as an autosampler and a system controller.

This communication describes a simple manual method for on-line precolumn derivatization using a Rheodyne type syringe loading sample injector.

\section{Experimental}

A Rheodyne Model 7125 syringe injector with a 20$\mathrm{mm}^{3}$ loop $(0.5 \mathrm{~mm}$ i.d.) was used for the derivatization of amino acids. Two pumps (JAsco 880-PU), a degasser (JASCO DG-3510), a solvent mixing module (JASCO 880-30), a fluorometer (JASCo FP-210) equipped with a $15-\mathrm{mm}^{3}$ flow cell and a recorder (Sic Chromatocorder 11) were used. A Shim-pack LC-ODS (particle size $10 \mu \mathrm{m}, 4.6 \mathrm{~mm}$ i.d. $\times 25 \mathrm{~cm}$ ) and a Shim-pack ODS guard column $(4.6 \mathrm{~mm}$ i.d. $\times 4 \mathrm{~cm}$, Shimadzu) were used.

OPA, MCE and 16 amino acids (arginine, histidine, lysine, tyrosine, tryptophan, phenylalanine, methionine, serine, threonine, leucine, isoleucine, valine, glutamic acid, aspartic acid, glycine and alanine) were obtained from Wako Pure Chemical Industries Ltd. The water was redistilled from a hard-glass vessel. The standard solutions of amino acid were prepared by dissolving amino acids in water to give a concentration of 100 $\mathrm{pmol} / \mathrm{mm}^{3}$ and diluted adequately. An OPA solution $\left(1 \mathrm{mg} / \mathrm{cm}^{3}\right.$ ) in $0.4 \mathrm{M}$ borate buffer ( $\mathrm{pH} 9.2$ ) was prepared; it was stable for at least ten days when kept in the dark at room temperature. Four microliters of MCE was added to $2 \mathrm{~cm}^{3}$ of the OPA solution. Fresh OPA/MCE solution was prepared each day.

The compositions of the mobile phases were as follows: solution A, acetate buffer (0.05 M, pH 6.0): $\mathrm{MeOH}$ : THF $=79: 20: 1$; solution $B$, acetate buffer $(0.05 \mathrm{M}, \mathrm{pH}$ 6.0): $\mathrm{MeOH}=20: 80$.

\section{Procedure}

With the injector valve in "Injection" position, flush out the needle port with $2-3 \mathrm{~cm}^{3}$ of the mobile phase using the needle port cleaner. Then turn the valve to "Load" position. Insert a syringe needle into the needle port and inject $3 \mathrm{~mm}^{3}$ of the OPA/MCE solution. Immediately insert another syringe and inject $3 \mathrm{~mm}^{3}$ of amino acid solution. Leave the syringe and the valve in "Load" position for $4 \mathrm{~min}$. After exactly $4 \mathrm{~min}$ turn the valve to "Injection" position. Then the derivative is fed into the column. Measure the fluorescence intensity by setting the excitation wavelength at $340 \mathrm{~nm}$ and the emission wavelength at $455 \mathrm{~nm}$.

\section{Results and Discussion}

Figure 1 shows the effect of derivatization time in the sample loop on the fluorescence intensity. The fluo-

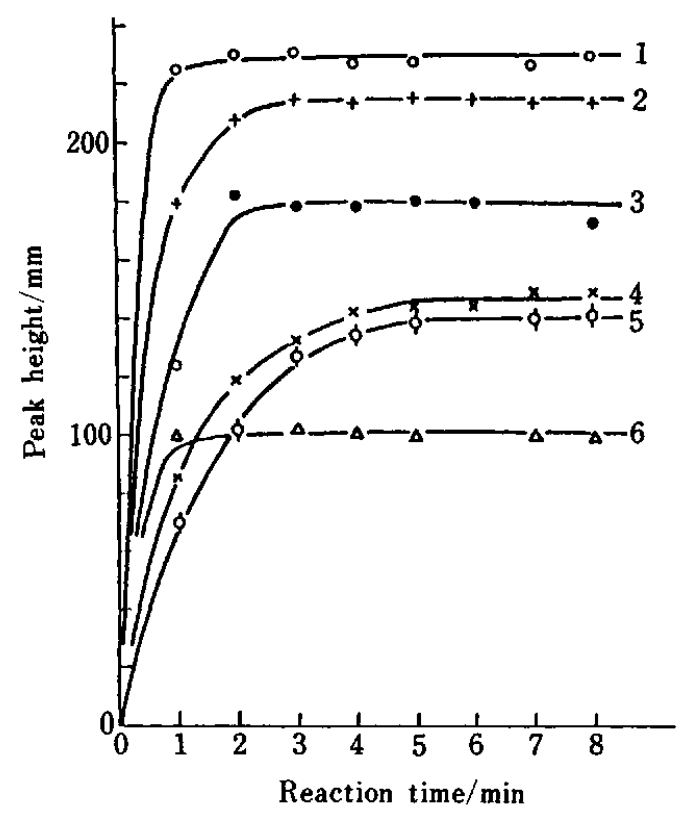

Fig. 1 Effect of reaction time on derivatization of amino acids with OPA/MCE reagent. Isocratic elution, $0.05 \mathrm{M}$ acetate buffer (pH 6.0): $\mathrm{MeOH}=50: 50$; sample $\left(50 \mathrm{pmol} / \mathrm{mm}^{3}\right)$; flow rate, $0.5 \mathrm{~cm}^{3} / \mathrm{min} ; 1$, serine; 2 , glutamic acid; 3 , arginine; 4 , aspartic acid; 5 , glycine; 6 , alanine. 


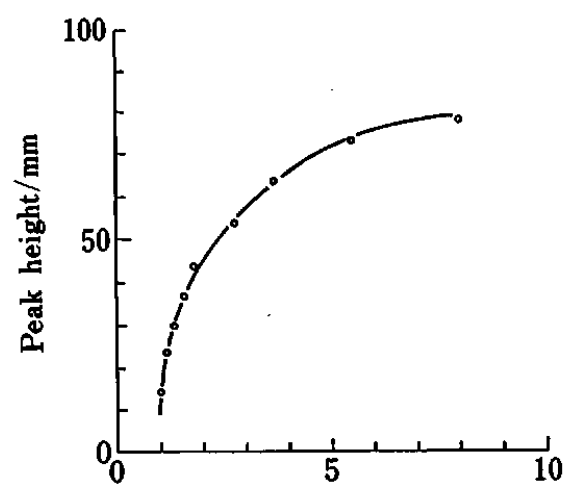

Amount of OPA/MCE solution $/ \mathrm{mm}^{3}$

Fig. 2 Effect of amount of OPA/MCE solution. Sample: serine, $20 \mathrm{pmol} / \mathrm{mm}^{3}$. Mobile phase and flow rate are as in Fig. 1.

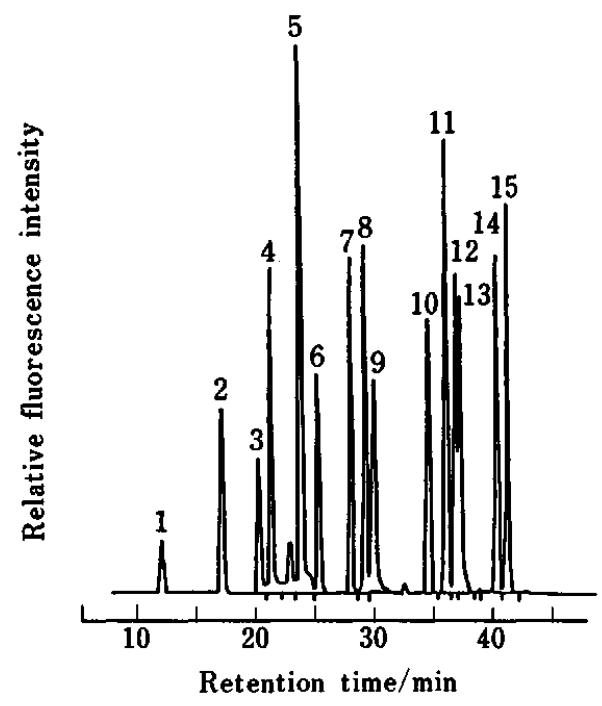

Fig. 3 Gradient elution of 15 amino acid derivatives. Mobile phase (solution $\mathrm{A} /$ solution $\mathrm{B}, \mathrm{v} / \mathrm{v}$ );

$\begin{array}{lrrrrr}\text { Time (min): } & 0 & 6 & 16 & 35 & 45 \\ \text { Solution A: } & 100 & 86 & 50 & 10 & 100 \\ \text { Solution B: } & 0 & 14 & 50 & 90 & 0\end{array}$

Flow rate, $0.5 \mathrm{~cm}^{3} / \mathrm{min}$; peaks: 1 , aspartic acid; 2 , glutamic acid; 3 , histidine; 4 , serine; 5 , arginine; 6 , threonine; 7 , glycine; 8 , tyrosine; 9 , alanine; 10 , tryptophan; 11 , methionine; 12 , valine; 13 , phenylalanine; 14 , isoleucine; 15 , leucine.

rescence intensity of the derivatives generally increased until 4 min after introducing OPA/MCE solution and amino acid solution to the sample loop. Thus, after exactly $4 \mathrm{~min}$, the sample was delivered into the column. Until at least $8 \mathrm{~min}$ the derivatives were stable in the sample loop. Calibration graphs were examined for five amino acids: serine, glutamic acid, arginine, aspartic acid, glycine and alanine. Good linearity of calibration graphs was obtained for $10-80 \mathrm{pmol} / \mathrm{mm}^{3}$ of each amino acid. The sensitivity of this method is

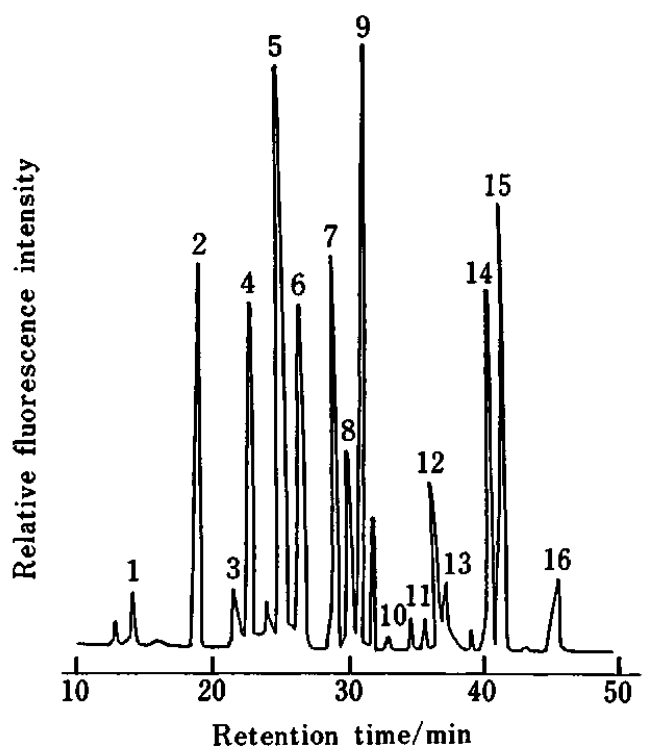

Fig. 4 Analysis of Japanese sake. Sake was diluted 100 times. Mobile phase and flow rate are as in Fig. 3. Peaks are as in Fig. 3 except for 16, lysine.

relatively high because no extra dispersion of sample occurs. The largest relative standard deviations of the peak height and the peak area were about $4 \%$ for six injections $\left(20 \mathrm{pmol} / \mathrm{mm}^{3}\right)$.

Figure 2 shows the effect of the amount of the OPA/MCE solution. The peak height increased with the amount of the reagent solution. Three microliters was employed to gain reasonable sensitivity.

A typical chromatogram of 15 standard amino acids using gradient run is shown in Fig. 3. When THF was not added to the mobile phase, histidine and serine, threonine and glycine, valine and phenylalanine were not completely resolved from each other. This method was successfully applied to the analysis of Japanese sake (Fig. 4).

By the proposed method, the derivatization and injection of sample can be carried out using simple and conventional devices, and the consumption of derivatization reagents is small. Therefore, this method would find practical use in many fields.

\section{References}

1. S. S. Simons and D. F. Johnson, J. Org. Chem., 43, 2886 (1978).

2. M. O. Fleury and D. V. Ashley, Anal. Biochem., 133, 330 (1983).

3. D. C. Turnell and J. D. H. Cooper, J. Auto. Chem., 5, 36 (1983).

4. J. D. H. Cooper, G. Ogden, J. McIntosh and D. C. Turnell, Anal. Biochem., 142, 98 (1984).

(Received May 26, 1989)

(Accepted June 16, 1989) 